\title{
FM jamming cancellation suppression based on noise floor estimation with dynamic threshold
}

\author{
Jie Bai ${ }^{1}$, Guohong $\mathrm{Wang}^{1}$,Min $\mathrm{Wu}^{2}$,Shichao Liu ${ }^{1}$, Yang Lin $^{1}$,Xin Tingting ${ }^{1}$ \\ ${ }^{1}$ Naval Aeronautics University, Yantai, China \\ ${ }^{2} 75841$ troop, Changsha, China
}

\begin{abstract}
Technique of FM jamming time domain cancellation suppression based on noise floor estimation with dynamic threshold is researched, by using the colored noise NLR filter estimation algorithm instead of the narrowband filtering algorithm, the problem that the target echo signal is lost in the process of jamming amplitude estimation is solved. Through the phase analysis of the jamming signal, the problem of jamming signal amplitude estimation in the background of echo signal is transformed into the problem of useful signals spectral estimation in colored noise background. Combined with the characteristics of the low frequency components of the useful signal, the traditional NLR filtering algorithm is improved by adding the dynamic threshold, so as to achieve the purpose of colored noise's non-invasive estimation, and then to obtain more accurate jamming signal amplitude estimation. The simulation results show that compared with the traditional jamming cancellation algorithm, this algorithm can improve the SJR of the echo signal better and the anti - jamming ability is stronger.
\end{abstract}

\section{Introduction}

FM jamming has advantages such as variable bandwidth and small amplitude dynamic range, because of which it has a wide range of applications in the field of radar confrontation. At present, scholars have done a lot of research on FM jamming. Literature [1] analyzes the signal, spectrum and the interference effect on pulse compression radar of the FM jamming; literature [2] introduces random differential theory, and studies the interference effect the FM jamming has on pulse compression filter. In the literature [3], the radar signal containing the FM jamming signal is decomposed in different frequency bands by multi-scale time-frequency analysis and reconstruction of the wavelet packet, and then the original signal is reconstructed to realize the interference suppression.

In those literatures, the generation and suppression of FM jamming are studied respectively, but the suppression algorithm of strong FM jamming is less. In response to this problem, the literature [4] proposed a noise cancellation interference suppression algorithm based on time domain cancellation, the mathematical model and the main parameters of FM jamming are discussed. The interference amplitude is estimated by logarithmic transformation and the jamming suppression is accomplished by reconstruction. However, when the jamming signal could not enter the receiver totally, the constant signal characteristics of the jamming signal is destroyed, thereby reducing the effectiveness of the jamming suppression algorithm. The literature [5] proposed the amplitude disturbance of the FM jamming suppression algorithm, through the echo signal phase demodulation and narrowband filtering to estimate the disturbance, and then complete the time domain cancellation. However, this algorithm has lost in the target echo signal, and is not conducive to the detection of the target. To solve this problem, in this paper, the jamming reduction algorithm is improved by combining with the noise floor estimation algorithm.

\section{Suppression of FM Jamming Based on Time Domain Cancellation}

\subsection{Jamming Cancellation Algorithm}

In the background of FM jamming, the radar received echo signal model expresses as:

$$
x(t)=s_{r}(t)+J_{F M}(t)+n(t)
$$

In formula (1), $s_{r}(t)$ and $n(t)$ represent target echo signal and Gaussian white noise. FM jamming with amplitude disturbance expresses as:

$$
J_{F M}(t)=\left(A_{j}+\zeta(t)\right) \exp \left(j \phi_{j}(t)\right)
$$

In formula (2), $\zeta(t)$ represents the amplitude disturbance term, suppose that the $\zeta(t)$ is a narrowband low frequency Gaussian random noise with mean of 0 . $\phi_{j}(t)$ is the instantaneous phase, subjecting to 0 mean random distribution, $\zeta(t)$ and $\phi_{j}(t)$ are independent of each other. 
In strong interference environment, the target echo signal and the noise signal are much weaker than the jamming signal, therefore, put the target echo signal and process noise into one, formula (2) can be simplified as:

$$
\begin{aligned}
x(t) & =s^{\prime}(t)+J_{F M}(t) \\
& =A^{\prime}(t) \exp \left(j \phi^{\prime}(t)\right)+\left(A_{j}+\zeta(t)\right) \exp \left(j \phi_{j}(t)\right)
\end{aligned}
$$

In formula (3), $A^{\prime}(t)$ and $\phi^{\prime}(t)$ represent the instantaneous amplitude and instantaneous phase of $s^{\prime}(t) \cdot A^{\prime}$ and $A_{j}$ have the following relationship:

$$
A^{\prime}(t)<<A_{j}
$$

For further transformation, formula (3) can be expressed as:

$$
x(t)=J_{F M}(t)\left(1+s_{1}(t)\right)
$$

Where,

$$
\begin{aligned}
s_{1}(t) & =\frac{s^{\prime}(t)}{J_{F M}(t)} \\
& =\frac{A^{\prime}(t)}{A_{j}+\zeta(t)} \exp \left(j\left(\phi^{\prime}(t)-\phi_{j}(t)\right)\right)
\end{aligned}
$$

Logarithmic transformation to both sides of formula(5), and use first order approximation of Taylor expansion, the following formula is got:

$$
\begin{aligned}
\ln (x(t)) & =\ln \left(J_{F M}(t)\right)+\ln \left(1+s_{1}(t)\right) \\
& =\ln \left(A_{j}+\zeta(t)\right)+j\left(\phi_{j}(t)-2 k \pi\right)+s_{1}(t)
\end{aligned}
$$

Where, $k=\bmod \left(\phi_{j}(t), 2 \pi\right)$.

The imaginary part of (7) is:

$$
\begin{aligned}
& I(t)=\operatorname{Im}[\ln (x(t))]=\phi_{j}(t)-2 k \pi+\operatorname{Im}\left[s_{1}(t)\right] \\
& \quad \text { For }\left|s_{1}(t)\right|<<1, I(t)=\operatorname{Im}[\ln (x(t))] \approx \phi_{j}(t)-2 k \pi
\end{aligned}
$$

can be got from formula (8), which is the phase estimation of jamming signal. The phase demodulation of the echo signal is carried out according to the interference instantaneous phase estimation:

$$
\begin{aligned}
y(t) & =x(t) \exp (-j \operatorname{Im}(\ln (x(t)))) \\
& =\left[s^{\prime}(t)+J_{F M}(t)\right] \exp (-j \operatorname{Im}(\ln (x(t)))) \\
& =s^{\prime}(t) \exp (-j \operatorname{Im}(\ln (x(t))))+\left(A_{j}+\zeta(t)\right) \exp \left[-j \operatorname{Im}\left(s_{1}(t)\right)\right]
\end{aligned}
$$

The jamming item is indicated by $\vartheta(t)$ :

$$
\begin{aligned}
\vartheta(t) & =\left(A_{j}+\zeta(t)\right) \exp \left[-j \operatorname{Im}\left(s_{1}(t)\right)\right] \\
& =\left(A_{j}+\zeta(t)\right) \exp [-j \kappa(t)]
\end{aligned}
$$

Where, $\kappa(t)$ is a random process that satisfies the following formula:

$$
|\kappa(t)|<<1
$$

Do approximation of Taylor expansion to formula (11):

$$
\begin{aligned}
\vartheta(t) & =\left(A_{j}+\zeta(t)\right) \exp [-j \kappa(t)] \\
& =\left(A_{j}+\zeta(t)\right)\left[1-j \kappa(t)-\frac{\kappa^{2}(t)}{2 !}+\cdots\right] \\
& =\left(A_{j}+\zeta(t)\right)+\left(A_{j}+\zeta(t)\right) o(\kappa(t))
\end{aligned}
$$

Because $|\kappa(t)|<<1$, the following formula holds:

$$
\begin{aligned}
\vartheta(t) & =\left(A_{j}+\zeta(t)\right) \exp [-j \kappa(t)] \\
& \approx\left(A_{j}+\zeta(t)\right)
\end{aligned}
$$

Therefore,

$$
\begin{aligned}
y(t) & =x(t) \exp (-j \operatorname{Im}(\ln (x(t)))) \\
& \approx s^{\prime}(t) \exp (-j \operatorname{Im}(\ln (x(t))))+\left(A_{j}+\zeta(t)\right)
\end{aligned}
$$

For $y(t)$, it can be seen as the sum of weak echo signal entry and strong jamming signal entry, among which, the echo signal is a broadband signal, while the jamming signal is a narrowband signal under the assumption. At the same time, because the echo signal and jamming signal are generated in different ways, so the power rate changes differently. Based on the above analysis, the echo signal term is regarded as non-Gaussian colored noise, and the jamming signal term is regarded as the target signal. The estimation problem of the jamming signal item can be converted into the detection problem of the target signal in the background of colored noise. To solve the problem: the noise floor of the input signal is first estimated and then the power spectrum of the target signal is corrected according to the estimation result.

\subsection{Noise Floor Estimation Algorithm Based on NLR Filter}

Let $S(n)$ be the input signal power spectrum, $Y(n)$ be the estimated noise base, the noise base estimation process based on NLR filtering is represented by the following recursion:

$$
\begin{gathered}
Y(k)=Y(k-1)+e(k) \\
e(k)= \begin{cases}S(k)-Y(k-1) & |S(k)-Y(k-1)|<\lambda \\
\lambda \operatorname{sgn}(S(k)-Y(k-1)) & \text { else }\end{cases}
\end{gathered}
$$

Where, $\lambda$ is the threshold, $\operatorname{sgn}(x)$ is the symbolic function. When $x<0, \operatorname{sgn}(x)=-1$, when $x>0$, $\operatorname{sgn}(x)=1$.

It can be seen from formula (16), when the power spectrum of the input signal changes slowly, the power spectrum difference between adjacent two moments is smaller than the threshold value, and the power spectrum value at the next time is directly equal to the input signal power spectrum, while when the power spectrum of the input signal changes rapidly, the power spectrum difference value of the adjacent two moments is greater than the threshold value, and the power spectrum value at the next time is increased by the smaller step size. It can be seen that the NLR filtering effect is closely related to the threshold, and the threshold changes with the bandwidth of the input signals. When the input signal's bandwidth is wide or includes signals in different bandwidth, the threshold selection of the NLR filtering algorithm will be contradictory. The threshold setting is too large, which will cause the noise power spectrum estimation value to rise faster, resulting in the estimation of the noise power spectrum. If the threshold 
setting is small, the noise base estimation The change step is small, resulting in noise power spectrum estimation.

\subsection{NLR Filtering Algorithm Based on Dynamic Threshold}

From the formula (14), it can be seen that in the background of this paper, the input signal is composed of the weaker broadband noise and the strong narrowband low frequency target signal, so the low frequency target signal in the input signal is very prominent, it is easy to get the start and end locations of the power spectrum of target signal. In order to achieve the lossless estimation of the noise base in the no-target signal band, it is necessary to select a large threshold to match the rate of change of the noise floor fluctuation. In the target signal band, improve the Traditional NLR Filtering Algorithm: the adjustment factors $a$ and $b$ are used to dynamically adjust the threshold value, $(a<1, b>1)$, so that in the target signal frequency band, the noise base can be more accurate non-destructive estimation, so as to obtain a more accurate target signal power spectrum correction results.

In the target signal band, the adjustment factor on the threshold of the correction process is as follows: when the value of $S(k)-Y(k-1)$ is greater than 0 for multiple times, it can be considered that this location is the start point of the target signal power spectrum, In this case, the threshold $\lambda$ is corrected to be:

$$
\lambda=\lambda a^{n}, a<1
$$

Where, $n$ is the number of times that $S(k)-Y(k-1)>0$ appears continually. Using the adjustment coefficient $a$ to gradually reduce the threshold to avoid that the noise floor is estimated higher than the true value. When the value of $S(k)-Y(k-1)$ is smaller than 0 for multiple times, it can be considered that this location is the end point of the target signal power spectrum, In this case, the threshold $\lambda$ is corrected to be:

$$
\lambda=\lambda b^{n}, b>1
$$

Where, $n$ is the number of times that $S(k)-Y(k-1)<0$ appears continually. Using the adjustment factor $b$ to gradually increase the threshold, the noise base estimate is rapidly decreased, approaching the true noise base as soon as possible.

The introduction of the adjustment coefficient solves the problem of noise base over-estimation and under-estimation caused by the fixed threshold. but at the same time, to make the filtering effect better in the whole frequency, the filtering threshold of the target signal band $\lambda_{s}$, the initial threshold value $\lambda_{j}$ and the adjustment coefficient $a$ and $b$ at the target signal band need determining

For the threshold $\lambda_{s}$ at the target signal band, in order to make the estimation algorithm match the change rate of the noise floor, select the maximum value of the adjacent time power spectrum difference as the threshold value:

$$
\lambda_{s}=\max \left[S_{s}(k+1)-S_{s}(k)\right], k=1,2 \cdots
$$

Where, $S_{s}(k)$ is the band of no target signal.

Since the NLR filtering algorithm is based on the difference between the input signal power spectrum and the noise floor estimation, the relationships between the power spectrum and the initial threshold, as well as the adjustment coefficient are analyzed at the target signal band. The initial threshold $\lambda_{j}$ is determined by using the average difference of the adjacent time of the input signal power spectrum, and the value of the adjustment coefficient $a, b$ are determined according to the selected initial threshold and the noise floor fluctuation.

It can be seen from a large number of simulation experiments that, take half of the average difference in the power spectrum of the adjacent time as the initial threshold, the estimated value of the noise base can be matched with the true value. So the calculation of the initial threshold is as follows:

$$
\lambda_{j}=\frac{1}{2} \frac{1}{N-1} \sum_{k=2}^{N} d(k)
$$

Where, $d(k)=S_{j}(k)-S_{j}(k-1), k=1,2 \ldots \quad, \quad S_{j}(k)$ indicates the target signal band.

Under the action of the adjustment coefficient $a$, the noise base estimation value gradually increases and eventually stabilizes. Due to the adjustment of the coefficient $a$, the maximum estimated value of the noise base is:

$$
\lambda+a \lambda+a^{2} \lambda+\cdots \quad: \lambda \frac{1}{1-a}
$$

Let the noise floor fluctuation of input signal may reach be $m \mathrm{~dB}$, then make

$$
\lambda \frac{1}{1-a}=m
$$

The value of the available adjustment factor $a$ is $1-\lambda / m$

Since the value of the maximum noise base fluctuation is not known precisely, or the value of the noise floor fluctuation changes with time due to the time-varying characteristics of the channel, the noise floor fluctuation is deviated from the real situation. When the estimated value is less than the true value, it may cause the estimated value of the noise floor from the true value. Therefore, if the undulation of the real noise could not be predicted, the noise floor can be selected to ensure no loss of the noise floor estimated value.

Under the action of the adjustment coefficient $b$, the noise floor estimate is rapidly decreased at the end of the power spectrum of the target signal to approximate the true value of the noise floor as soon as possible, which has little effect on the estimation of the noise base, and any optional adjustment coefficient that satisfies $b>1$ is ok

At the same time, in order to solve the problem of asymmetry of target signal power spectrum caused by adjustment coefficients $a$ and $b$ in different values. The 
input signal power spectrum is arranged in reverse order and the dynamic threshold NLR filter is re-estimated, get the average of both sides. By means of the mean value, the spectral peak shape of the target signal can be substantially preserved, and the signal-to-noise ratio loss caused by the excessive noise floor estimation can be reduced. The estimated value of the power spectrum of the target signal can be obtained by subtracting the estimated value of the noise spectrum of the input signal power spectrum.

NLR filtering algorithm based on dynamic threshold makes it possible to estimate the power spectrum of the interfering signal more accurately. Compared with the narrowband filter estimation results of the interfering signal in the literature [5], this algorithm not only can get more accurate interference signal estimation, and can effectively reduce the loss of the echo signal. The estimation of the interference signal term $\varsigma(t)$ can be obtained by NLR filtering, reference formula (2), the reconstruction of FM jamming signal with amplitude disturbance is expressed as followed:

$$
\hat{J}_{F M}(t)=\varsigma(t) \exp (j I(t))
$$

Where $I(t)$ is the instantaneous phase estimation of the interfering signal obtained from formula (8). Eliminate the reconstructed interference signal from the echo signal $x(t)$ :

$$
\begin{aligned}
\hat{s}^{\prime}(t) & =x(t)-\hat{J}_{F M}(t) \\
& =s^{\prime}(t)+J_{F M}(t)-\hat{J}_{F M}(t)
\end{aligned}
$$

Through the time domain cancellation algorithm, in the more complete preservation of the echo signal under the premise of most of the FM jamming signal energy is eliminated, which is conducive to the subsequent detection of the target.

\section{Simulation results and analysis}

Simulation parameter settings: LFM signal pulse width $T_{p}=30 \mu \mathrm{s}$, the carrier frequency $f_{0}=4 \mathrm{MHz}$, bandwidth $B=2 M H z$. The carrier frequency of FM jamming signal $f_{j}=4 \mathrm{MHz}$, modulation index $m_{f e}=1000$, the process noise is Gaussian white noise. Radar sampling frequency $f_{s}=20 \mathrm{MHz}$, FM jamming signal bandwidth $B_{j}=0.3$ (normalized frequency), $\mathrm{SJR}$ is $-30 d B$, SNR is $-10 d B$. Under this simulation condition, the validity of the algorithm is verified.

\subsection{Verification of Dynamic Threshold NLR Filtering Algorithm}

Determine the threshold $\lambda_{s}$, the initial threshold $\lambda_{j}$, and the value of the adjustment factor $a$ according to formula (23), (24) and (26), adjustment factor $b=1.5$. In the band with the jamming signal, the threshold $\lambda$ is dynamically adjusted according to formula (21) and (22). Figure 1 shows the results of the power spectrum estimation of the echo signal of the traditional NLR filter algorithm and the correction of the power spectrum of the interference signal under the condition of the simulation parameters. Figure 2 shows the results of the power spectrum estimation of the echo signal of the dynamic threshold NLR filter algorithm and the correction result of the power spectrum of the interference signal under the simulation parameters.
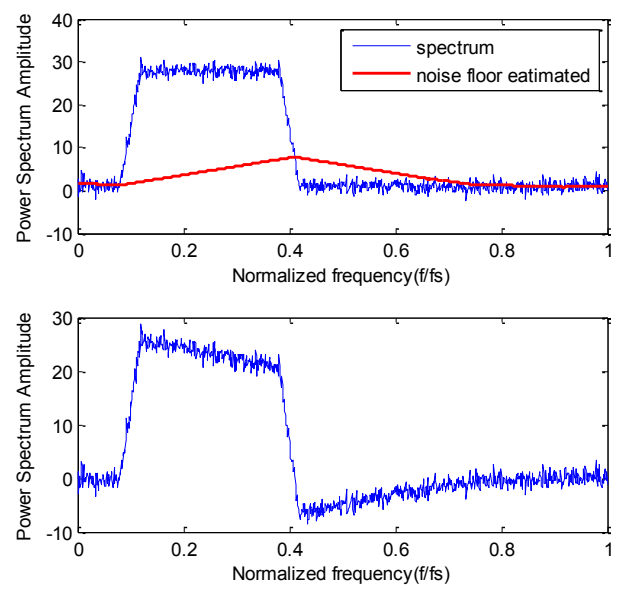

Figure.1 Traditional NLR filtering algorithm
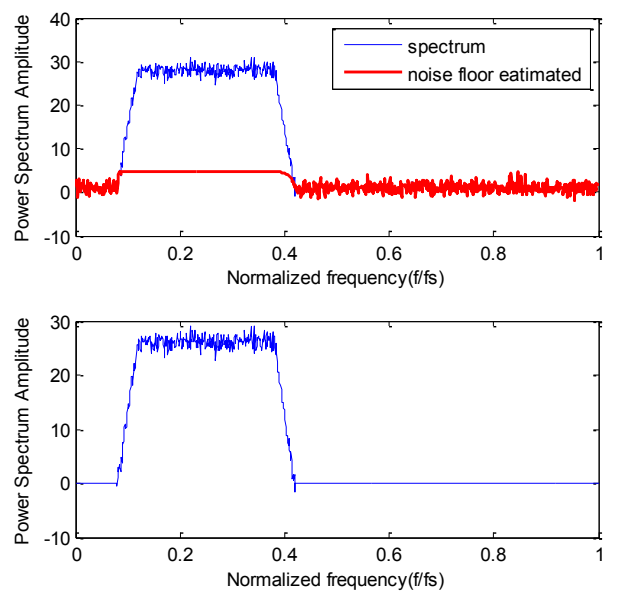

Figure.2 NLR filtering algorithm with dynamic threshold

In figure 1 and figure 2 , the abscissa indicates the normalized frequency, and the ordinate represents the power spectral amplitude value. The red lines in figure 1 (a) and figure 2 (a) show the power spectrum estimation of the echo signal term. It can be seen from the comparison of figure 1 and figure 2 that the traditional NLR filtering algorithm has a large error in the power spectrum estimation result of the interference signal. At the end of the power spectrum of the interfering signal, the estimation result is difficult to converge quickly and the dynamic threshold NLR filtering algorithm can realize the accurate estimation of the power spectrum of the interference signal in the whole frequency band, and it will not cause the loss of the echo signal term.

\subsection{Verification of validity of cancellation algorithm}


According to the dynamic threshold NLR filtering results, the interference signal is reconstructed and canceled in the time domain. Figure 3 and Figure 4 show the pulse compression results of the echo signal before and after the cancellation.

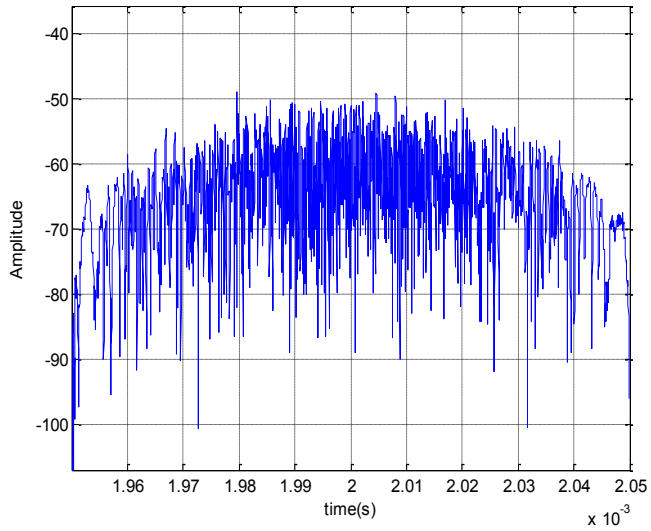

Figure. 3 The pulse compression before the interference cancellation

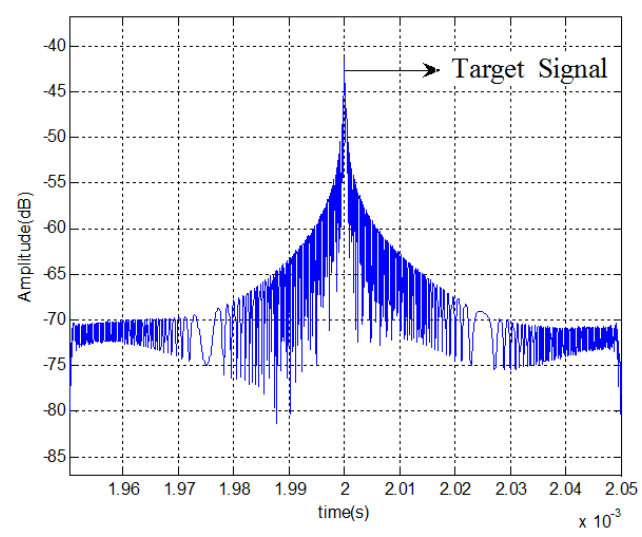

Figure.4 The pulse compression before and after the interference cancellation

The abscissa represents the time and the ordinate represents the signal amplitude in figure 3 and figure 4 . As can be seen from Figure 3 and Figure 4, the time domain interference cancellation algorithm eliminates most of the noise Energy, in order to achieve a strong FM jamming environment for the effective detection of the target signal.

\subsection{Algorithm comparison}

Change the FM jamming amplitude disturbance signal bandwidth range of 0 to 0.5 (normalized frequency), where the bandwidth of 0 means that the interference amplitude is not received disturbance. Figure 5 and figure 6 show the signal-to-interference ratio (SJR) curves of the literature [5] and the algorithm of this paper in different perturbation signal bandwidth.

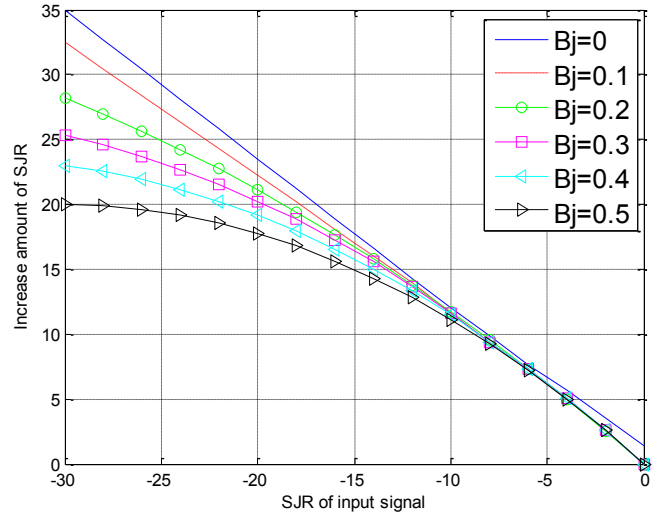

Figure.5 SJR increased amount of literature [5]

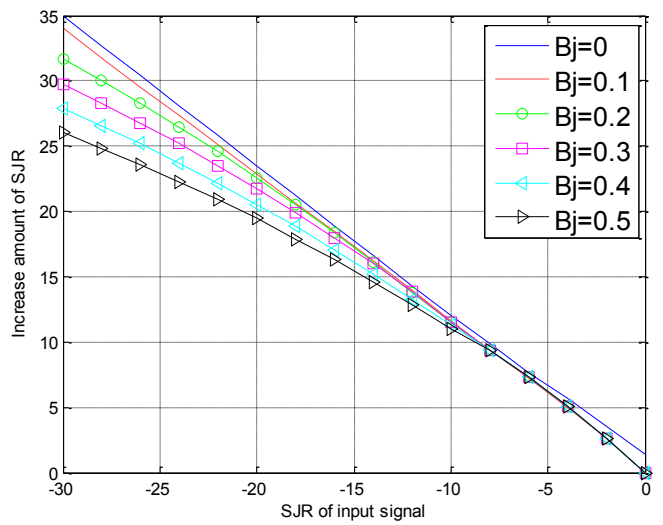

Figure.6 SJR increased amount of this paper

The abscissa indicates the signal-to-noise ratio of the echo signal, and the ordinate represents the increase in the signal-to-noise ratio of the output signal in figure 5 and figure 6 . It can be seen from figure 5 that the narrowband filtering algorithm in literature [5] not only could not achieve the accurate estimation of the interference signal term, but also causes the loss of the target echo signal, and the greater the bandwidth of the disturbance signal, therefore, the SJR increases with the widening of the disturbance signal bandwidth under the same conditions. As can be seen from figure 6, since the algorithm proposed in this chapter does not cause loss to the target echo signal, the change in SJR increases with the disturbance signal bandwidth compared with the literature [5], due to the interference signal Estimate of the error. Through the comparison of figure 5 and figure 6 , we can see that the algorithm in this paper solves the shortcoming of the loss of echo signal in literature [5] algorithm. In the strong FM jamming environment, the improvement of the signal-to-interference ratio is superior to that of the literature [5].

\section{Conclusions}

In this paper, the problem of loss of the target echo is solved by using the NLR filtering algorithm instead of the narrowband filter algorithm. According to the application background of NLR filtering, this paper combines the low frequency components of the input signal, The traditional NLR filtering algorithm is improved to complete the nondestructive estimation of the echo signal term, and the estimation of the 
interference signal term is corrected to achieve the better reconstruction effect of the interference signal. It can be seen from the simulation results that this algorithm has better interference suppression ability in strong noise FM interference environment.

\section{References}

1. Y.S. Li, H.Lv, and Y.J.Liu. Research on Noise FM Jamming and its Realization on FPGA, Electronic Science and Technology, 28,(2015), 139-141.

2. Q.Q. Sun, K.Zheng, X.Y.Cao, Jamming Mechanism of Wideband Noise FM Signals to Pulise Compression, RADAR \& ECM, 36,(2016), 30-34.

3. Z.G. Liu, W.Wang. J.Y.Zhang., Research on shipboard radar anti noise frequency modulation jamming based on envelope filtering, SHIP SCIENCE AND TECHNOLOGY, 31,(2009),70-72.

4. H.L. Dong, S.F. Li, D.L.Luo. Simulation Analysis of Anti-noise FM Jamming Algorithm Based on Time-domain Cancellation, Journal of Air Force Early Warning Academy, 4,(2013), 249-251.

5. D.P.Du, Research on Radar Suppression Interference Suppression Algorithm. Chengdu: University of Electronic Science and Technology, 2008, 83-87. 